\title{
Suppression of the primary resonance vibrations of a forced nonlinear system using a dynamic vibration absorber
}

\author{
J.C. Ji, N. Zhang \\ Faculty of Engineering, University of Technology, Sydney \\ PO Box 123, Broadway, NSW 2007, Australia
}

\begin{abstract}
In a single-degree-of-freedom weakly nonlinear oscillator subjected to periodic external excitation, a small-amplitude excitation may produce a relatively large-amplitude response under primary resonance conditions. Jump and hysteresis phenomena that result from saddle-node bifurcations may occur in the steady-state response of the forced nonlinear oscillator. A simple mass-spring-damper vibration absorber is thus employed to suppress the nonlinear vibrations of the forced nonlinear oscillator for the primary resonance conditions. The values of the spring stiffness and mass of the vibration absorber are significantly lower than their counterpart of the forced nonlinear oscillator. Vibrational energy of the forced nonlinear oscillator is transferred to the attached light mass through linked spring and damper. As a result, the nonlinear vibrations of the forced oscillator are greatly reduced and the vibrations of the absorber are significant. The method of multiple scales is used to obtain the averaged equations that determine the amplitude and phases of the first-order approximate solutions to primary resonance vibrations of the forced nonlinear oscillator. Illustrative examples are given to show the effectiveness of the dynamic vibration absorber for suppressing primary resonance vibrations. The effects of the linked spring and damper and the attached mass on the reduction of nonlinear vibrations are studied with the help of frequency response curves, the attenuation ratio of response amplitude, and the desensitisation ratio of the critical amplitude of excitation.
\end{abstract}




\section{Introduction}

In a forced single-degree-of-freedom weakly nonlinear system, nonlinear resonances may occur if the linearized natural frequency of the system and the frequency of an external excitation satisfy a certain relationship. A small-amplitude excitation may produce a relatively large-amplitude response under primary resonance conditions when the forcing frequency is in the neighbourhood of the linearized natural frequency. Additionally, the steady-state forced response of the nonlinear system may exhibit nonlinear dynamic behaviours including saddle-node bifurcations, jump and hysteresis phenomena [1]. These behaviours along with large-amplitude resonant vibrations are undesirable in many applications because they can result in unacceptable levels of vibration and discontinuous dynamic behaviour. The nonlinear vibrations and jump phenomena should thus be attenuated by appropriate approaches from the perspective of vibration control and disaster prevention.

Over the past decade, active control methods have been developed to suppress the nonlinear resonance vibrations of weakly nonlinear systems with parametric or external excitations. These methods include time-delayed feedback control [2-6], a linear-plusnonlinear feedback control $[7,8]$, and a nonlinear parametric feedback control [9]. The application of such feedback control schemes to the control of nonlinear vibrations and bifurcations does not add an extra degree-of-freedom system into the nonlinear systems to be controlled. Introduction of an 'auxiliary system' into the existing linear structures to be controlled has also been considered using nonlinear coupling and internal resonances between the auxiliary system and the linear primary structure. An internal resonance control technique has been developed by creating a linear secondorder controller coupled to a linear vibration system via quadratic terms [10-12]. The controller was implemented by making use of the saturation phenomenon which may exist in a weakly non-linear system with quadratic nonlinearities and under one-to-two internal resonance between two linearized natural frequencies [1]. Under internal resonances, the quadratic nonlinear terms act as a bridge for energy exchange between the linear system and the controller. It should be noted that the use of active controllers for attenuation of vibrations is not feasible in many applications, for reasons including cost or the need of an independent energy supply. A passive control approach is an alternative under these circumstances [13]. Additionally, a passive vibration control system may be required as a back-up to prevent complete disaster in the event of the failure of active control methods. 
In the context of the passive vibration control of linear mechanical systems, one of the well developed approaches for reducing vibration levels is to add a secondary linear oscillator to the existing linear system or structure. This secondary oscillator, commonly called a dynamic vibration absorber $[14,15]$, may be a simple mass-spring-damper system attached at a single point of a linear mechanical system or structure. The main purpose of adding the secondary oscillator is to move the resonant frequency of the mechanical system away from the operating frequency of the vibratory force. The original single-degree-of-freedom system becomes a two-degree-of-freedom system with two resonant frequencies, neither of which will coincide with the operating frequency. The dynamic vibration absorber is usually tuned in such a way that two natural frequencies of the resulting two-degree-of-freedom linear system are away from the excitation (operating) frequency [14-17]. When a dynamic vibration absorber is incorporated into a linear mechanical system, vibrations of the mechanical system at its operating frequency can be reduced to negligible proportions and no peak in amplitudes of the response is reached. Instead, two peaks appear at frequencies below and above the fundamental frequency of the original system.

Dynamic vibration absorbers have found extensive applications in reducing the amplitudes of vibrations of linear systems excited near a resonant frequency [14-20]. Linear dynamic vibration absorbers are an effective way of attenuating vibrations of the primary linear system provided that the operational frequency of the primary linear system is constant. It was found that better performance of dynamic vibration absorbers can be achieved by introducing nonlinear absorber springs. It has been shown that the nonlinear vibration absorber incorporating a nonlinear coupling spring element could offer performance advantages in both narrow- and broad-band applications over its linear counterpart [21-25]. But, unfortunately, the presence of nonlinearities may introduce dynamic instabilities and result in amplification rather than reduction of the vibration amplitudes [26-28]. Such situations can generally occur in two cases. The first case is when the nonlinear vibration absorber is tuned in such a way that the desired operating frequency is approximately the mean of the two linearized natural frequencies of the system $[26,27]$. Thus a combinational resonance may result in near-periodic vibrations having large amplitudes. The other case is when the two linearized natural frequencies of the resultant system are under one-to-one internal resonance conditions [28]. Loss of stability of the periodic response and quasiperiodic oscillations with much higher amplitudes may happen for the primary 
resonance response of the nonlinear system under certain combinations of system parameters.

In addition to the use of either linear or nonlinear vibration absorbers to suppress the vibrations of linear systems, linear vibration absorbers can also be applicable to controlling the nonlinear vibrations of nonlinear mechanical systems. An experimental study by Bonsel, Fey and Nijmeijer [29] showed that a linear dynamic vibration absorber is capable of suppressing the first harmonic resonance as well as super- and sub-harmonic resonances of a piecewise linear beam.

The main purpose of the present paper is to suppress the primary resonance vibrations of a weakly nonlinear system with a periodic excitation using a linear vibration absorber. The linear vibration absorber referred to here is a mass that is relatively light in comparison with the mass of the nonlinear primary system and is attached to the nonlinear primary system by a linear spring and a linear damper (also called coupling). The damping coefficient and the spring stiffness of the absorber are much lower than their counterpart, as such the vibration absorber can be considered as a small attachment to the nonlinear primary system. The addition of an absorber to the nonlinear primary system (one-degree-of-freedom weakly nonlinear system) results in a new two degree-of-freedom weakly nonlinear system. The characteristics of the nonlinear primary system attached by the linear absorber change only slightly in terms of the values of its new linearizied natural frequency, damping coefficient and frequency interval for primary resonance, because the vibration absorber is a small attachment and does not contribute significantly to the change of these parameters (linear stiffness and damping coefficient). Two ratios, namely attenuation ratio and desensitisation ratio, will be defined in the present paper to indicate the effectiveness of the linear absorber in suppressing the primary resonance vibrations. The attenuation ratio will be defined by the ratio of the maximum amplitude of vibrations of the nonlinear primary system after and before adding the linear vibration absorber under a given value of the amplitude of excitation. The desensitisation ratio will be given by the ratio of the critical values of the amplitude of external excitations presented in the nonlinear primary system after and before the linear vibration absorber is attached. The critical value of the excitation amplitude refers to here as a certain value of external excitation that results in the occurrence of saddle-node bifurcations and jump phenomena in the frequency-response curve. Below this critical value, the frequency-response curve of the primary resonance vibrations does not show saddle-node bifurcations (and jump phenomena) and will exhibit saddle-node bifurcations and jump phenomena if the 
amplitude of excitation exceeds the critical value. It will be shown that the linear vibration absorber is effective in attenuating the primary resonance vibrations of the nonlinear primary system. The underlying mechanism is that the addition of linear absorber to the nonlinear primary system modifies the coefficients in the averaged equations (as will be shown in Section 3 ) that determine the amplitude and phase of the first-order approximate solution and thereby modifying the frequency-response curve of the nonlinear primary system under primary resonance conditions.

The present paper is organised into six sections. Section 2 presents the mathematical modelling of the vibrations of a nonlinear primary system attached by a linear vibration absorber. Perturbation analysis is performed in Section 3 to obtain the averaged equations that determine the amplitude and phase of the first-order approximate solution of a two-degree-of-freedom nonlinear system. Section 4 introduces the attenuation ratio and desensitisation ratio to indicate the effectiveness of linear vibration absorber. Illustrative examples are presented in Section 5 and conclusion is given in Section 6.

\section{Mathematical Modelling}

It is assumed here that a single degree-of-freedom weakly nonlinear system may be described as one which consists of a mass subjected to a periodic excitation. The mass $m_{1}$ is attached to a rigid boundary through a viscous damper and a spring of linear-plus-nonlinear characteristic, as shown in Figure 1. A significantly lighter mass $m_{2}$ (in comparison with the main mass $m_{1}$ ), which will be referred to here as a small attachment, is connected to the nonlinear system (also called nonlinear primary system) through a massless damper and a massless spring. The addition of the small attachment to the nonlinear primary system results in a new two degree-of-freedom nonlinear system.

By applying Newton's second law of motion, two equations of motion for the new system composed of the nonlinear primary system incorporated by a small attachment may be written as:

$$
\begin{aligned}
& m_{1} \ddot{x}_{1}=-k_{1} x_{1}-k_{2} x_{1}^{3}-c_{1} \dot{x}_{1}+k_{3}\left(x_{2}-x_{1}\right)+c_{2}\left(\dot{x}_{2}-\dot{x}_{1}\right)+f_{0} \cos (\Omega t), \\
& m_{2} \ddot{x}_{2}=-k_{3}\left(x_{2}-x_{1}\right)-c_{2}\left(\dot{x}_{2}-\dot{x}_{1}\right),
\end{aligned}
$$


where $m_{1}$ denotes the mass of the nonlinear primary system and $m_{2}$ the mass of the small attachment. $k_{1}, k_{2}$ and $c_{1}$ represent the linear, nonlinear stiffness and damping coefficient in relation to mass $m_{1}$, respectively. The coupling stiffness and damping coefficient are $k_{3}$ and $c_{2}$. The displacements of the nonlinear primary system and the small attachment, as shown in Figure 1, are denoted by $x_{1}$ and $x_{2}$. An overdot indicates the differentiation with respect to time $t$.

Dividing $m_{1}$ on both sides of the first equation and dividing $m_{2}$ on both sides of the second equation and then rewriting the resultant equations yields the following equations:

$$
\begin{gathered}
\ddot{x}_{1}+\mu_{1} \dot{x}_{1}+\omega_{1}^{2} x_{1}-m \mu_{2} \dot{x}_{2}-m \omega_{2}^{2} x_{2}+\alpha x_{1}^{3}=f \cos (\Omega t), \\
\ddot{x}_{2}+\mu_{2}\left(\dot{x}_{2}-\dot{x}_{1}\right)+\omega_{2}^{2}\left(x_{2}-x_{1}\right)=0,
\end{gathered}
$$

where $\mu_{1}=\left(c_{1}+c_{2}\right) / m_{1}=\mu_{10}+m \mu_{2}, \quad \omega_{1}^{2}=\left(k_{1}+k_{3}\right) / m_{1}=\omega_{10}^{2}+m \omega_{2}^{2}, \quad m=m_{2} / m_{1}$, $\mu_{2}=c_{2} / m_{2}, \omega_{2}^{2}=k_{3} / m_{2}, \alpha=k_{2} / m_{1}, f=f_{0} / m_{1}, \mu_{10}=c_{1} / m_{1}, \omega_{10}^{2}=k_{1} / m_{1}$. Here, parameters $\mu_{10} \omega_{10}$ are used to represent the damping coefficient and the linearized natural frequency of the weakly nonlinear oscillator alone.

Equation (2) can be interpreted in the context of nonlinear oscillations as a two degreeof-freedom weakly nonlinear system subjected to a periodic excitation. The forced oscillations of a two-degree-of-freedom nonlinear system having cubic nonlinearities have been studied by many researchers [1, 28]. The attention of these studies has focused on the case of internal resonances when $\omega_{1} \approx \omega_{2}$ or $\omega_{2} \approx 3 \omega_{1}$. Specifically, Nayfeh and Mook [1] considered the forced oscillations of cubically nonlinear systems without linear coupling terms under internal resonances $\omega_{2} \approx 3 \omega_{1}$. Natsiavas [28] studied the steady-state oscillations and stability of the nonlinear system having cubic nonlinearities under one-to-one internal resonances (i.e. $\omega_{1} \approx \omega_{2}$ ). It was shown that the presence of one-to-one internal resonances in the nonlinear system of dynamic vibration absorber may result in instability of the periodic response and quasi-periodic oscillations with much higher amplitudes. The mathematical modelling considered in the present paper represents a two degree-of-freedom nonlinear system that consists of a one-degree-of-freedom weakly nonlinear oscillator linearly coupled with a linear oscillator. The focus of the present paper is on the use of linear vibration absorber to suppress the primary resonance response of a weakly nonlinear oscillator in the 
absence of internal resonances, rather than steady-state solutions and stability of the system under one-to-one internal resonances. The natural frequency of the linear absorber alone and the linearized natural frequency of the nonlinear oscillator are not under internal resonances including one-to-one and three-to-one resonances.

It is noted that nonlinear resonances may occur in the forced response of the resultant system when the forcing frequency $\Omega$ and the linearized natural frequency $\omega_{1}$ satisfy a certain relationship. The primary resonance response of the nonlinear system given by equation (2) will be discussed in subsequent section using a perturbation method, as the closed form of the solutions to equation (2) cannot be found analytically.

\section{Perturbation analysis}

A brief discussion on the order of the coefficients in Equation (2) is necessary before performing perturbation analysis. It should be mentioned that the main purpose of the present research is to investigate the suppression of the nonlinear vibrations of a nonlinear primary oscillator using a small attachment without adversely affecting the performance of the nonlinear primary oscillator. The connection between the nonlinear primary system and small attached mass is via linear damper and spring. The small attached mass and the damping and spring stiffness of coupling can be considered as a perturbation to the nonlinear primary oscillator, in a sense that the nonlinear primary system is weakly coupled with the small attachment. As a result, the linear stiffness and mass of the nonlinear primary oscillator should be much larger than the stiffness of the linked spring and the mass of the small attachment. For the attachment, though its stiffness and mass are small in comparison with those of the nonlinear primary system, the linear stiffness of the attachment is comparable with its mass and thus is assumed to be leading terms in equation (2b). In particular, all damping terms and nonlinear term are assumed to be small and in the order of $O(\varepsilon)$ in equation (2a) and the damping term is considered to be in the order of $O(\varepsilon)$ in equation (2b). Then the perturbation analysis will result in the leading linear terms in the first-order equations, the small linear and nonlinear terms in the second-order equations. As the values of the coupled stiffness and damping for the nonlinear primary oscillator is much smaller than those of its own stiffness and damping, the property of the nonlinear primary system does not change significantly.

On the basis of the above discussions on the order of the coefficients, equation (2) can be rewritten as 


$$
\begin{gathered}
\ddot{x}_{1}+\varepsilon \mu_{1} \dot{x}_{1}+\omega_{1}^{2} x_{1}-\varepsilon m \mu_{2} \dot{x}_{2}-\varepsilon m \omega_{2}^{2} x_{2}+\varepsilon \alpha x_{1}^{3}=f \cos (\Omega t), \\
\ddot{x}_{2}+\varepsilon \mu_{2} \dot{x}_{2}+\omega_{2}^{2} x_{2}=\varepsilon \mu_{2} \dot{x}_{1}+\omega_{2}^{2} x_{1},
\end{gathered}
$$

where $\varepsilon$ is a dimensionless parameter with $\varepsilon<<1$, the coefficients of the damping term and nonlinear term, $\mu_{1}, \mu_{2}$ and $\alpha$ in equation (2) have been re-scaled in terms of $\mu_{1}=\varepsilon \bar{\mu}_{1}, \mu_{2}=\varepsilon \bar{\mu}_{2}$, and $\alpha=\varepsilon \bar{\alpha}$, and the overbars in $\bar{\mu}_{1}, \bar{\mu}_{2}$ and $\bar{\alpha}$ have been removed for brevity. The amplitude of the excitation has been re-scaled in terms of $f=\varepsilon \bar{f}$ to account for the primary resonances and the overbar in $\bar{f}$ has been removed for the sake of brevity. Equation (3) can be regarded as a weakly nonlinear system with an external excitation being coupled by a linear system.

It is noted that two terms: $\varepsilon \mu_{2} \dot{X}_{1}$ and $\omega_{2}^{2} x_{1}$ that appear on the right hand side of equation (3b), act as external excitations to the attached oscillator (linear vibration absorber). The majority of vibrational energy of the nonlinear primary system is then transferred to the linear absorber through the coupling terms between the nonlinear primary oscillator and vibration absorber. The vibrational energy flowing from nonlinear primary system to absorber results in a reduction of primary resonance vibration of the nonlinear primary system.

The method of multiple scales is employed to obtain a set of four averaged equations that determine the amplitudes and phases of the steady state solutions on a slow scale [1]. For the sake of simplicity, only the first-order approximate solutions will be sought in subsequent analysis. It is assumed that the solutions of equation (3) in the neighbourhood of the trivial equilibrium are represented by an expansion of the form:

$$
\begin{aligned}
& x_{1}(t ; \varepsilon)=x_{10}\left(T_{0}, T_{1}\right)+\varepsilon x_{11}\left(T_{0}, T_{1}\right)+O\left(\varepsilon^{2}\right), \\
& x_{2}(t ; \varepsilon)=x_{20}\left(T_{0}, T_{1}\right)+\varepsilon x_{21}\left(T_{0}, T_{1}\right)+O\left(\varepsilon^{2}\right),
\end{aligned}
$$

where $\varepsilon$ is a non-dimensional small parameter, $T_{0}=t$ is a fast scale associated with changes occurring at the frequencies $\omega_{1}$ and $\Omega$, and $T_{1}=\varepsilon t$ is slow scale associated with modulations in the amplitude and phase caused by the non-linearity, damping and resonances. The derivatives of $x_{1}$ and $x_{2}$ with respect to $t$ then become expansions in terms of partial derivatives with respect to $T_{0}$ and $T_{1}$ given by:

$$
\frac{d}{d t}=\frac{d T_{0}}{d t} \frac{\partial}{\partial T_{0}}+\frac{d T_{1}}{d t} \frac{\partial}{\partial T_{1}}=D_{0}+\varepsilon D_{1}
$$




$$
\frac{d^{2}}{d t^{2}}=\left(\frac{\partial^{2}}{\partial T_{0}^{2}}+2 \varepsilon \frac{\partial^{2}}{\partial T_{0} \partial T_{1}}+O\left(\varepsilon^{2}\right)\right)=D_{0}^{2}+2 \varepsilon D_{0} D_{1}+O\left(\varepsilon^{2}\right) .
$$

It should be noted that equation $(3 b)$ is a linear differential equation and thus its solution can be expressed in a closed form as long as solutions $x_{1}$ and $\dot{x}_{1}$ are available. The solution to equation ( $3 b)$ consists of general and particular solutions depending on the solutions $x_{1}$ and $\dot{x}_{1}$ that can be obtained from Equation (3a). As defined by equation (4), the solution $x_{1}$ cannot be expressed in a closed form. Here it has thus been assumed that the solution $x_{2}$ is also expressed as an approximate solution comprising of two parts.

Substituting the approximate solutions (4) into equation (3), taking into consideration the new multiple independent variables of time, and then balancing the like powers of $\varepsilon$, results in the following ordered perturbation equations:

$$
\begin{gathered}
\varepsilon^{0} \quad D_{0}^{2} x_{10}+\omega_{1}^{2} x_{10}=0, \quad D_{0}^{2} x_{20}+\omega_{2}^{2} x_{20}=\omega_{2}^{2} x_{10} \\
\varepsilon \quad D_{0}^{2} x_{11}+\omega_{1}^{2} x_{11}=-2 D_{0} D_{1} x_{10}-\mu_{1} D_{0} x_{10}+m \mu_{2} D_{0} x_{20}+m \omega_{2}^{2} x_{20}-\alpha x_{10}^{3}+f \cos \left(\Omega T_{0}\right) \\
D_{0}^{2} x_{21}+\omega_{2}^{2} x_{21}=-2 D_{0} D_{1} x_{20}-\mu_{2} D_{0} x_{20}+\mu_{2} D_{0} x_{10}+\omega_{2}^{2} x_{11}
\end{gathered}
$$

The general solutions to equation (5) can be expressed in complex form as

$$
\begin{aligned}
& x_{10}=A\left(T_{1}\right) \exp \left(i \omega_{1} T_{0}\right)+c c, \\
& x_{20}=F_{0} A\left(T_{1}\right) \exp \left(i \omega_{1} T_{0}\right)+B\left(T_{1}\right) \exp \left(i \omega_{2} T_{0}\right)+c c,
\end{aligned}
$$

where $A\left(T_{1}\right)$ and $B\left(T_{1}\right)$ are an arbitrary function at this level of approximation. $F_{0}=1 /\left(1-\omega_{1}^{2} / \omega_{2}^{2}\right)$, and $c c$ stands for the complex conjugate of the preceding terms. $A\left(T_{1}\right)$ and $B\left(T_{1}\right)$ will be determined by imposing the solvability conditions at the next level of approximations.

For the case of primary resonances, the forcing frequency is assumed to be almost equal to the linearized natural frequency of the nonlinear primary system according to

$$
\Omega=\omega_{1}+\varepsilon \sigma,
$$

where $\sigma$ is an external detuning parameter to express the nearness of $\Omega$ to $\omega_{1}$.

Substituting solution (7) into equation (6) yields

$$
\begin{aligned}
D_{0}^{2} x_{11}+\omega_{1}^{2} x_{11}= & -i 2 \omega_{1}\left(D_{1} A\right) \exp \left(i \omega_{1} T_{0}\right)+\frac{1}{2} f \exp \left(i \omega_{1} T_{0}+i \sigma T_{1}\right)-\alpha A^{3} \exp \left(i 3 \omega_{1} T_{0}\right)+ \\
& \left(m \omega_{2}^{2} F_{0} A-3 \alpha A^{2} \bar{A}-i \mu_{1} \omega_{1} A+i m \mu_{2} \omega_{1} F_{0} A\right) \exp \left(i \omega_{1} T_{0}\right)+
\end{aligned}
$$




$$
\begin{gathered}
\left(m \omega_{2}^{2} B+i m \mu_{2} \omega_{2} B\right) \exp \left(i \omega_{2} T_{0}\right)+c C, \\
D_{0}^{2} x_{21}+\omega_{2}^{2} x_{21}=-i 2 \omega_{2}\left(D_{1} B\right) \exp \left(i \omega_{2} T_{0}\right)+\omega_{2}^{2} x_{11}-i \mu_{2} \omega_{2} B \exp \left(i \omega_{2} T_{0}\right)+ \\
{\left[i \mu_{2} \omega_{1} A-i \mu_{2} \omega_{1} F_{0} A-2 i \omega_{1} F_{0}\left(D_{1} A\right)\right] \exp \left(i \omega_{1} T_{0}\right)+c c}
\end{gathered}
$$

where $\bar{A}\left(T_{1}\right)$ is the complex conjugate of $A\left(T_{1}\right)$. For the sake of brevity, $A\left(T_{1}\right)$, $B\left(T_{1}\right)$ and $\bar{A}\left(T_{1}\right)$ have been expressed by $A, B$ and $\bar{A}$, respectively.

Solution $x_{21}$ can be obtained from equation (9b) only after solution $x_{11}$ to equation (9a) is available. It should be made aware that only the term with argument $\exp \left( \pm i \omega_{2} T_{0}\right)$ in solution $x_{11}$ will make contributions to the secular terms in equations (9b). In eliminating the terms that lead to secular terms from equation (9b), the particular solution $x_{11}$ of equation (9a) can be written as

$$
x_{11}=K B \exp \left(i \omega_{2} T_{0}\right)+\mathrm{NST}+c c,
$$

where $K=\left(m \omega_{2}^{2}+i m \mu_{2} \omega_{2}\right) /\left(\omega_{1}^{2}-\omega_{2}^{2}\right)$, and NST stands for the terms that do not produce secular terms in seeking solution $x_{21}$.

Then, eliminating the terms that lead to secular terms from equation (9) yields

$$
\begin{aligned}
& \frac{1}{2} f \exp \left(i \sigma T_{1}\right)+\left(m \omega_{2}^{2} F_{0}-i \mu_{1} \omega_{1}+i m \mu_{2} \omega_{1} F_{0}\right) A-3 \alpha A^{2} \bar{A}-2 i \omega_{1} D_{1} A=0, \\
& \omega_{2}^{2} K B-i \mu_{2} \omega_{2} B-2 i \omega_{2} D_{1} B=0 .
\end{aligned}
$$

The functions $A$ and $B$ (i.e. $A\left(T_{1}\right)$ and $B\left(T_{1}\right)$ ) can be expressed in the polar form as

$$
A=\frac{1}{2} a\left(T_{1}\right) \exp \left[i \beta\left(T_{1}\right)\right], \quad B=\frac{1}{2} b\left(T_{1}\right) \exp \left[i \theta\left(T_{1}\right)\right],
$$

where $a\left(T_{1}\right), b\left(T_{1}\right), \beta\left(T_{1}\right)$ and $\theta\left(T_{1}\right)$ are real functions of time $T_{1}$.

Substituting equation (12) into equation (11) and then separating real and imaginary parts gives rise to

$$
\begin{aligned}
& \dot{a}=g_{11} a-e \sin (\gamma), \\
& a \dot{\gamma}=-\left(\sigma+g_{210}\right) a+g_{22} a^{3}-e \cos (\gamma), \\
& \dot{b}=h_{11} b, \\
& b \dot{\theta}=h_{21} b,
\end{aligned}
$$

where for notation purpose four functions $a\left(T_{1}\right), b\left(T_{1}\right), \beta\left(T_{1}\right)$ and $\theta\left(T_{1}\right)$ have been expressed by $a, b, \beta$ and $\theta$, respectively, $\gamma=\beta-\sigma T_{1}, g_{11}=\left(-\mu_{1}+m \mu_{2} F_{0}\right) / 2$, 
$e=f /\left(2 \omega_{1}\right), \quad g_{210}=m \omega_{2}^{2} F_{0} /\left(2 \omega_{1}\right), \quad g_{22}=3 \alpha /\left(8 \omega_{1}\right), \quad h_{11}=-\mu_{2}\left(1+m F_{0}\right) / 2$, $h_{21}=m \omega_{2} F_{0} / 2$.

The steady-state solutions to the primary resonance response can be studied by finding the solutions to the first three algebraic equations which can be obtained by letting $\dot{a}=\dot{b}=0$ and $\dot{\gamma}=0$ in equation (13). Elimination of the trigonometric terms in the first two algebraic equations gives rise to

$$
\begin{aligned}
& g_{11}^{2} a^{2}+\left(-\sigma-g_{210}+g_{22} a^{2}\right)^{2} a^{2}=e^{2}, \\
& h_{11} b=0 .
\end{aligned}
$$

This is the so-called frequency response equation. Real positive solutions of this equation that can be obtained numerically may lead to the frequency-response curve. It is easy to notice that the two equations are not coupled and the solution to the second equation is $b=0$. Equation (14) indicates that the amplitude of the first-order approximate solution to the free-oscillation term of the vibration absorber admits trivial solutions only. The response of the absorber consists of only forced vibrations resulting from the nonlinear primary system. The amplitude of the first-order approximate solution of the nonlinear primary system is determined by the first equation only, which is of the similar form to that of Duffing oscillator but with modified coefficients $g_{11}$ and $g_{22}$. The addition of a small attachment can thus change the amplitude of the firstorder approximate solution and the frequency-response curve. The stability of the solutions can be examined by computing the eigenvalues of the Jacobian matrix corresponding to the first three equations of equation (13). It is found that three eignenvalues determining the stability of the steady-state solutions are given by $\lambda_{1,2}=g_{11} \pm \sqrt{g_{22}^{2} a^{4}-\left(-\sigma-g_{210}+2 g_{22} a^{2}\right)^{2}}, \quad \lambda_{3}=h_{11}$. The steady-state solutions that can be obtained numerically from Equation (14) are stable only if the three eigenvalues have negative real parts.

Generally speaking, the frequency-response curve may exhibit saddle-node bifurcations, jump phenomena, and the coexistence of multiple solutions, when the coefficients in the first equation of (14) satisfy certain conditions. The saddle-node bifurcation points are at the locations of vertical tangency of the frequency response curve. Differentiation of the first equation of equation (15) implicitly with respect to $a^{2}$ and setting $d \sigma / d a^{2}=0$ leads to the condition:

$$
\left(g_{22} a^{2}-\sigma-g_{210}\right)^{2}+2 g_{22}\left(g_{22} a^{2}-\sigma-g_{210}\right) a^{2}+g_{11}^{2}=0
$$


with solutions being $\sigma_{ \pm}=2 g_{22} a^{2}-g_{210} \pm \sqrt{g_{22}^{2} a^{4}-g_{11}^{2}}$. For $g_{22}^{2} a^{4}>g_{11}^{2}$, there exists an interval $\sigma_{-}<\sigma<\sigma_{+}$in which three real solutions $a$ of equation (14) exist. In the limit $g_{22}^{2} a^{4}=g_{11}^{2}$, this interval shrinks to the point $\sigma=2 g_{22} a^{2}-g_{210}$, indicating no jump phenomena occurring in the frequency-response curve. The critical amplitude of excitation (denoted by $e_{\text {crit }}$ ) that can be obtained from Equation (14) is written as $e_{\text {crit }}=\sqrt{2\left|g_{11}^{3} / g_{22}\right|}$. For $e<e_{\text {crit }}$, there is only one solution for the amplitude $a$ in the neighbourhood of the primary resonances, while for $e>e_{\text {crit }}$, there are three solutions for the amplitude $a$ in the interval $\sigma_{-}<\sigma<\sigma_{+}$.

The peak amplitude (denoted by $a_{p}$ ) of the forced response for the nonlinear primary system with attached vibration absorber can also be obtained from equation (14) and is given by $a_{p}=e /\left|g_{11}\right|$. The peak amplitude is the maximum amplitude of the primary resonance vibrations under a given set of external excitations.

\section{Attenuation ratio and desensitisation ratio}

For the purpose of comparison, the equation of motion for the nonlinear primary oscillator without attached mass (see Figure 1) can be written as:

$$
\ddot{x}_{1}+\mu_{10} \dot{x}_{1}+\omega_{10}^{2} x_{1}+\alpha x_{1}^{3}=f \cos (\Omega t),
$$

where $\Omega=\omega_{10}+\varepsilon \sigma_{0}, \mu_{10}=c_{1} / m_{1}, \omega_{10}^{2}=k_{1} / m_{1}$, as introduced after equation (2) in Section 2.

The frequency-response equation is then given by:

$$
\left(\frac{\mu_{10}}{2}\right)^{2} a_{0}^{2}+\left(-\sigma_{0}+g_{220} a_{0}^{2}\right)^{2} a_{0}^{2}=e_{0}^{2}
$$

where $a_{0}$ denotes the amplitude of the primary resonance response of the nonlinear primary system without attachment, $e_{0}=f /\left(2 \omega_{10}\right), g_{220}=3 \alpha /\left(8 \omega_{10}\right)$.

The corresponding critical amplitude of excitation for the nonlinear primary oscillator alone, denoted by $e_{0 \text { crit }}$, is given by $e_{0 \text { crit }}=\sqrt{\mu_{10}^{3} /\left(4\left|g_{220}\right|\right)}$. Similarly, the corresponding peak amplitude of the forced response for the nonlinear primary oscillator alone, denoted by $a_{0 p}$, is found to be $a_{0 p}=2 e_{0} / \mu_{0}$. 
The performance of the vibration absorber on the reduction of nonlinear vibrations cannot be studied using a similar procedure to that for the linear system by discussing the ratio of response amplitude and the amplitude of excitation because the response amplitude cannot be found analytically for a nonlinear system (see equation (14)). Additionally, at certain frequencies of excitation, the nonlinear primary system may have two stable responses depending on the initial conditions of the system. Therefore a different method has to be developed here to study the performance of vibration absorber. In suppressing the primary resonance vibrations of the nonlinear primary oscillator, the performance of the vibration absorber will be examined in the present paper by defining two ratios, namely, the attenuation ratio of the peak amplitude of the primary resonance response, and the desensitisation ratio of the critical amplitude of the external excitation.

The attenuation ratio of the peak amplitude of primary resonance response is defined by the ratio of the peak amplitude of primary resonance vibrations of the nonlinear primary system with and without the attachment. By this definition the attenuation ratio, denoted by $R$, can be expressed as

$$
R=\frac{a_{p}}{a_{0 p}}=\left(\frac{e}{\left|g_{11}\right|}\right) /\left(\frac{2 e_{0}}{\mu_{10}}\right)=\frac{\mu_{10} \omega_{10}}{\left|-\mu_{1}+m \mu_{2} F_{0}\right| \omega_{1}}=\frac{\mu_{10} \omega_{10}}{\left|-\mu_{10}-m \mu_{2}+m \mu_{2} F_{0}\right| \omega_{1}} .
$$

As can be seen from the definition given by equation (18), under a fixed value of the amplitude of excitation, a small value of the attenuation ratio $R$ indicates a large reduction in the nonlinear vibrations of the nonlinear primary system. Given the fact that the damping coefficients $\left(\mu_{10}, \mu_{1}, \mu_{2}\right)$, mass ratio $(m)$, and the linearized natural frequencies $\left(\omega_{10}, \omega_{1}\right)$ are positive in values, it is easy to note that parameter $F_{0}$ should be negative in order to achieve a large reduction ratio and get better performance of vibration suppression. An analysis of the value of $F_{0}=1 /\left(1-\omega_{1}^{2} / \omega_{2}^{2}\right)$ reveals that $F_{0}<0$ for $\omega_{1}>\omega_{2}$, and $F_{0}>1$ for $\omega_{1}<\omega_{2}$. A negative $F_{0}$ requires the linearized natural frequencies of the nonlinear primary system and vibration absorber satisfying $\omega_{1}>\omega_{2}$, indicating the natural frequency of the vibration absorber should be smaller than the new linearized natural frequency of the nonlinear primary system attached by the vibration absorber.

The desensitisation ratio (denoted by $E$ ) of the critical amplitudes of the excitation is defined by the ratio of the critical amplitudes of the excitations presented in the 
nonlinear primary system with and without attached vibration absorber, which is given by

$$
E=\frac{e_{\text {crit }}}{e_{0 \text { crit }}}=\frac{\sqrt{\left(-\mu_{1}+m \mu_{2} F_{0}\right)^{3} \omega_{1}}}{\sqrt{\mu_{10}^{3} \omega_{10}}}=\frac{\sqrt{\left(-\mu_{10}-m \mu_{2}+m \mu_{2} F_{0}\right)^{3} \omega_{1}}}{\sqrt{\mu_{10}^{3} \omega_{10}}} .
$$

A large desensitisation ratio $(E>1)$ corresponds to a large critical amplitude of the excitation. A larger desensitisation ratio indicates that saddle-node bifurcations and jump phenomena will be eliminated in the primary resonance response of the nonlinear primary system with the attachment of vibration absorber, for a given value of the amplitude of excitation where saddle-node bifurcations and jump phenomena can appear in the frequency-response curve of the nonlinear primary system alone. The vibration absorber cannot only suppress the nonlinear vibrations but can also eliminate the saddle-node bifurcations which otherwise appear in the primary resonance response of the nonlinear primary system before the linear vibration absorber is attached. Given that damping coefficients, mass ratio, and frequencies $\left(\omega_{1}>\omega_{10}>0\right)$ are all positive in values, it is easy to note that a negative $F_{0}$ results in a larger $E$, which means better performance can be achieved by ensuring two linearized natural frequencies satisfying $\omega_{1}>\omega_{2}$. A negative value of $F_{0}$ ensures that $E>1$. The smaller $F_{0}$, the larger $E$.

It should be mentioned that a negative $F_{0}$ will lead to a better performance of reduction of primary resonance vibrations. The smaller negative $F_{0}$, the better performance of vibration reduction. As given in the equation $F_{0}=1 /\left(1-\omega_{1}^{2} / \omega_{2}^{2}\right)$, a much smaller $F_{0}$ can be obtained when $\omega_{2}$ is approaching to $\omega_{1}$ under $\omega_{1}>\omega_{2}$. However, numerical simulations have suggested the primary resonance vibrations cannot always be attenuated but rather magnified under certain combination of system parameters and initial conditions if $\omega_{2}$ is too close to $\omega_{1}$. As such, the internal resonances of one-toone type occur in the primary resonance response of the two degree-of-freedom nonlinear system consisting of the nonlinear primary system and vibration absorber. The interaction of one-to-one internal resonances would lead to an increase of the amplitudes of primary resonance vibrations under certain combinations of system parameters. The numerical results confirmed the theoretical predictions of the nonlinear interaction of nonlinear systems given in the literature. For example, Natsiavas [28] studied steady-state oscillations and stability of nonlinear dynamic 
vibration absorbers under one-to-one internal resonances (i.e. $\omega_{1} \approx \omega_{2}$ ) and found the loss of stability of the periodic response and the onset of quasi-periodic oscillations with much higher amplitudes. The vibrations of the two-degree-of-freedom nonlinear system under one-to-one internal resonances are outside the scope of the present paper. Though no analytical evidence is available in the literature, it is fairly safe to assume that $\omega_{2}$ should be less than $0.9 \omega_{1}$ in order to avoid the one-to-one internal resonances. For the purpose of vibration reduction, the frequency $\omega_{2}$ should be away from frequency $\omega_{1}$ but can still ensure a smaller negative $F_{0}$.

In suppressing the vibration of a linear system, the addition of a secondary massspring-damper system to the main linear system will create a combined system with two resonant frequencies. Resonances will occur if the excitation frequency is close to one of the natural frequencies and thus the combined system exhibits two peaks in the response [13-17]. For a weakly nonlinear oscillator attached by a linear vibration absorber, as discussed in the present paper, it is natural to assume that an extra peak will appear in the forced response resulting from the introduction of a new resonant frequency (i.e. the natural frequency of the absorber). It is expected that a peak in amplitude will appear around this frequency. Mathematically the combined system of the nonlinear primary oscillator and linear absorber can be considered as a synthesis of a weakly nonlinear oscillator and a linear oscillator through linear coupling. It should be noted that for a single-degree-of-freedom nonlinear oscillator under non-resonant hard excitations, the steady-state response consists of the forced solution only, as in the linear case [1]. In this regard, the effect of weekly nonlinear terms on the steadystate response can be neglected as long as the frequency of the excitation $\Omega$ is away $\omega_{1}, \frac{1}{3} \omega_{1}$, and $3 \omega_{1}$, which correspond to the primary resonances, super-harmonic resonances and sub-harmonic resonances, respectively. In the absence of internal resonances the steady-state motions of the combined system in the neighbourhood of the absorber frequency are linear and can be obtained from the corresponding linearized system.

It is assumed that the steady-state solutions to the corresponding linearized equation of equation (2) have the form:

$$
\begin{aligned}
& x_{1}=X_{11} \cos (\Omega t)+X_{12} \sin (\Omega t) ; \\
& x_{2}=X_{21} \cos (\Omega t)+X_{22} \sin (\Omega t) .
\end{aligned}
$$


The four unknown constants $X_{11}, X_{12}, X_{21}, X_{22}$ can be obtained by substituting equation (20) into equation (2) and by using Cramer's method. Substitution of equation (20) into equation (2) results in a system of four linear equations expressed in matrix form as

$$
\text { Ay }=p
$$

where $\mathrm{A}$ is a $4 \times 4$ matrix and the vector $y=\left(X_{11}, X_{12}, X_{21}, X_{22}\right)^{T}$ is the column vector of the variables. The solutions are given by

$$
y_{i}=\operatorname{det}\left(A_{i}\right) / \operatorname{det}(A) \quad i=1,2,3,4
$$

where $A_{i}$ is the matrix formed by replacing the ith column of $A$ by the column vector $p$, and the elements of matrix $A$ and vector $p$ are not given here for the sake of brevity.

\section{Illustrative examples and discussion}

This section presents illustrative examples to show the effectiveness of the linear vibration absorber for suppressing the nonlinear vibrations of the nonlinear oscillator under primary resonance conditions. The performance of linear vibration absorber on attenuation of nonlinear vibrations will be interpreted by the frequency-response curves, time histories of the forced response, the attenuation ratio of the maximum amplitudes of vibrations and the desensitisation ratio of critical amplitudes of the excitation of the nonlinear primary system before and after the absorber is attached.

Numerical simulations have been performed under the following values of the system parameters: $\quad m_{1}=10.0 \mathrm{~kg}, \quad m_{2}=0.6 \mathrm{~kg}, \quad c_{1}=0.1 \mathrm{Ns} / \mathrm{m}, \quad c_{2}=0.08 \mathrm{Ns} / \mathrm{m}$,

$k_{1}=44.0 \mathrm{~N} / \mathrm{m}, \quad k_{2}=8.0 \mathrm{~N} / \mathrm{m}^{3}, \quad k_{3}=2.0 \mathrm{~N} / \mathrm{m}$, unless otherwise specified. This combination of system parameters indicates that the mass ratio is $6 \%$ (i.e., the quotient of $m_{2} / m_{1}, m=0.06$ ) and the coupling stiffness is approximately $4.55 \%$ (which is obtained by the quotient of $k_{3} / k_{1}$ ) of the linear stiffness of the nonlinear primary system. This set of system parameters confirms a small mass attachment to the nonlinear primary system. The linearized natural frequencies of the nonlinear primary system before and after being attached by the vibration absorber are found to be approximately $\omega_{10}=2.0976 \mathrm{rad} / \mathrm{s}, \omega_{1}=2.1448 \mathrm{rad} / \mathrm{s}$, and the natural frequency of the vibration absorber be $\omega_{2}=1.8257 \mathrm{rad} / \mathrm{s}$. The linearized natural frequencies of the nonlinear primary system before and after the addition of vibration absorber change slightly, only at approximately $2.19 \%$. The selection of the parameters of linear 
vibration absorber made in the present paper is thus distinct from the one for controlling the linear vibrations of linear systems in the sense that for controlling linear vibrations, the natural frequencies of the resulting system composed of the linear system attached by vibration absorber are designed to be away from the excitation frequency. For the nonlinear system considered in the present paper, due to its distinct nature in primary resonances from the dynamics of linear system, there is no need to shift the linearized natural frequency of the nonlinear primary system away from the excitation frequency. The nonlinear vibrations of the nonlinear oscillator under primary resonance conditions can be significantly reduced by adding a small attachment, which is expected to be feasible in practical applications.

It is noted from the definitions of attenuation ratio and desensitisation ratio that the nonlinear stiffness of the nonlinear primary system has no effect on these two ratios as the parameter $\alpha$ is not included in the expressions of the two ratios. The absorber mass, stiffness and damping of coupling have significant effects on these two ratios. Figure 2 shows the variations of the attenuation ratio and desensitisation ratio with the damping, stiffness and mass of absorber. For fixed stiffness and mass of the absorber, increase of absorber damping $c_{2}$ leads to an increase of desensitisation ratio and a decrease of attenuation ratio, as shown in Figure 2a. As can be seen from equations (18) and (19), the parameter $\mu_{2}$ that corresponds to the absorber damping $c_{2}$ is involved in the term $\left(-\mu_{10}-m \mu_{2}+m \mu_{2} F_{0}\right)$, where the parameters $\mu_{10}, m, \mu_{2}$ are positive and $F_{0}$ is negative, thereby the term $\left(-\mu_{10}-m \mu_{2}+m \mu_{2} F_{0}\right)$ will be negative. An analysis of the two ratios by taking derivatives with respect to $\mu_{2}$ indicates that there is no optimal value of $\mu_{2}$ for a minimum value of attenuation ratio $R$ and a maximum value of desensitisation ratio $E$. This suggests that there is no optimal value of the absorber damping for attenuation of the nonlinear vibrations of the nonlinear oscillator, which is distinct from the suppression of vibrations of linear systems. For linear systems, an optimal value of the absorber damping can be found. A larger value of coupling damping results in a better performance of vibration reduction. The ratio of the linearizied natural frequencies of the resultant system, defined by $\psi=\omega_{2} / \omega_{1}$, is $\psi=0.8512$ for the curves corresponding to $k_{3}=2.0 \mathrm{~N} / \mathrm{m}$ and $\psi=0.7372$ for the curves corresponding to $k_{3}=1.5 \mathrm{~N} / \mathrm{m}$. Better performance of vibration reduction can be enhanced by a larger ratio of natural frequencies, as shown by the curves corresponding to $k_{3}=2.0 \mathrm{~N} / \mathrm{m}$ in Figure $2 \mathrm{a}$. 
For fixed damping and mass of the absorber, the desensitisation ratio increases and the attenuation ratio decreases as the stiffness of the absorber spring $k_{3}$ increases, as shown in Figure $2 \mathrm{~b}$. This is because an increase of absorber stiffness $k_{3}$ will increase the natural frequency $\omega_{2}$ of the vibration absorber, thereby increasing the ratio of the two linearized natural frequencies $\psi$. A larger ratio $\psi$ will lead to a smaller negative value of $F_{0}$, and the smaller negative value of $F_{0}$ will result in a smaller value of the attenuation ratio and a larger value of the desensitisation ratio. The ratio of the two linearized natural frequencies $\psi$ increases its value from 0.3002 to 0.7735 for the curves related to $m_{2}=1.0 \mathrm{~kg}$ and from 0.3356 to 0.8648 for the curves related to $m_{2}=0.8 \mathrm{~kg}$. A higher value of stiffness $k_{3}$ will lead to a better performance of vibration suppression, in essence, a higher value of frequency ratio $\psi$ will result in better performance of vibration attenuation. On the contrary, for fixed damping and stiffness of vibration absorber, the desensitisation ratio decreases and the attenuation ratio increases with an increase of the absorber mass, as shown in Figure $2 \mathrm{c}$. The underlying reason for this is that the natural frequency $\omega_{2}$ decreases with an increase of absorber mass $m_{2}$ under a constant stiffness $k_{3}$. The frequency ratio $\psi$ decreases its value from 0.8891 to 0.6287 for the curves corresponding to $k_{3}=2.0 \mathrm{~N} / \mathrm{m}$ and from 0.8559 to 0.5475 for the curves related to $k_{3}=1.5 \mathrm{~N} / \mathrm{m}$, which leads to a larger negative $F_{0}$ with an increase of the absorber mass $m_{2}$. This indicates that a small absorber mass $m_{2}$ leads to a better performance of vibration attenuation.

As indicated in Equations (18) and (19), the attenuation ratio is inversely proportional to the term $\left|-\mu_{1}+m \mu_{2} F_{0}\right|$ while the desensitisation ratio is cubically proportional to the term. A larger value of $\left|-\mu_{1}+m \mu_{2} F_{0}\right|$ means a better performance of vibration reduction, which requires a smaller negative $F_{0}$ for given values of damping coefficients and mass ratio. Recall from Section 3 that $F_{0}=1 /\left(1-\omega_{1}^{2} / \omega_{2}^{2}\right)$, being inversely proportional to the ratio of the square of the two linearized natural frequencies, $\psi=\omega_{2} / \omega_{1}$. It is fairly safe to suggest that vibration absorber should be designed in such a way that the frequency ratio $\psi$ is a certain value out of an interval 
of 0.7 to 0.9 , which corresponds to $F_{0}$ being a certain value of the region between 0.961 and -4.263 .

The performance of a vibration absorber on attenuation of nonlinear vibrations of nonlinear oscillator can be clearly demonstrated with the help of frequency-response curves. Figure 3 shows the frequency-response curves of the nonlinear primary system before and after the addition of the vibration absorber for the amplitude of excitation $f=0.42 \mathrm{~N}$, which are obtained by perturbation analysis. The horizontal axis represents an interval of external detuning $\sigma \in[-0.2,0.2] \mathrm{rad} / \mathrm{s}$, which corresponds to a small interval of forcing frequency $\Omega \in[1.9448,2.3448] \mathrm{rad} / \mathrm{s}$. Without adding the absorber, the peak amplitude of the nonlinear primary system is $0.75521 \mathrm{~cm}$ and saddle-node bifurcations occur in the frequency-response curve. In the interval $\sigma \in[-0.02,0.06] \mathrm{rad} / \mathrm{s}$, two stable solutions coexist with a unstable solution in between. Jump-up phenomenon happens at $\sigma=-0.02 \mathrm{rad} / \mathrm{s}$ when decreasing forcing frequency from $\sigma=0.2 \mathrm{rad} / \mathrm{s}$, and jump-down phenomenon occurs at $\sigma=0.06 \mathrm{rad} / \mathrm{s}$ when increasing forcing frequency from $\sigma=-0.2 \mathrm{rad} / \mathrm{s}$. After adding the absorber to the nonlinear primary system, the peak amplitude of the nonlinear system has been greatly reduced to $0.022796 \mathrm{~cm}$. The interval of the multiple coexisting solutions disappears and the jump phenomena are eliminated. The primary resonance vibrations of the nonlinear primary system have been significantly attenuated. As shown in Figure 3 , the frequencies at which the amplitudes of primary resonance vibrations reach their maximum have shifted from $\sigma=0.06 \mathrm{rad} / \mathrm{s}$ for the nonlinear primary system alone to $\sigma=0.155 \mathrm{rad} / \mathrm{s}$ for the nonlinear primary system with absorber. In terms of the frequency of excitation, the maximum amplitudes of primary resonance vibrations occur at $\Omega=2.2048 \mathrm{rad} / \mathrm{s}$ for the nonlinear primary system without absorber, and at $\Omega=2.2998 \mathrm{rad} / \mathrm{s}$ for the nonlinear primary system with vibration absorber.

Figure 4a shows the frequency-response curves for a large interval of the frequency of excitation $\Omega \in[1.2,2.8] \mathrm{rad} / \mathrm{s}$ of the nonlinear oscillator before and after adding absorber. Dashed and solid lines are used to represent the amplitudes of stable solutions of the nonlinear oscillator alone and of the nonlinear oscillator with vibration absorber attached, respectively. In the neighbourhood of primary resonances, the amplitude of nonlinear vibrations of the nonlinear primary oscillator has been greatly attenuated by adding the linear vibration absorber. The vibration of the nonlinear oscillator with attached vibration absorber is not zero, but its amplitude is very small in 
comparing with the amplitude of vibrations of the nonlinear oscillator alone. Outside of the interval of frequency $\Omega \in[1.76,2.19] \mathrm{rad} / \mathrm{s}$, the addition of a linear vibration absorber has slightly increased the amplitude of vibrations of the nonlinear primary oscillator except in the neighbourhood of two new linearized resonant frequencies. Therefore the performance of the absorber can be regarded as satisfactory. There are two resonant peaks appearing on the frequency-response curve of the nonlinear primary oscillator with absorber attached, which correspond to two resonant frequencies of the combined system. One is the natural frequency of the vibration absorber alone and the other is the new linearized natural frequency of the nonlinear primary oscillator incorporated with the vibration absorber. The peak at the lower frequency results from the resonances at the natural frequency of absorber and the peak at the higher frequency corresponds to the primary resonance response at the new linearized natural frequency of the nonlinear primary oscillator. The comparison of perturbation analysis (analytical predictions) and numerical integration is also shown in Figure 4a where circles represent analytical predictions on the amplitudes of nonlinear vibrations in the neighbourhood of primary resonances at $\omega_{1}$. Only small differences between the approximate and numerical integration solutions are found. The first-order approximate solutions match well with the numerical integration solutions. While the first-order approximate solutions obtained using the method of multiple scales give slightly larger values than the numerical integration solutions. The discrepancies are caused by the first-order truncation of the expansion solution. A more accurate approximation could be obtained if an additional term of the second order is included in the approximate solution, but seems unnecessary as the first-order approximations are already good representations of the primary resonance response.

Figure $4 \mathrm{~b}$ shows the frequency-response curves of the nonlinear oscillator with vibration absorber attached under different damping coefficients of the absorber. Increase of absorber damping leads to reduction of peak amplitude at resonant frequencies. Figure 4c shows the variation of the amplitude of vibrations of nonlinear oscillator attached by absorber under different nonlinear stiffness of the nonlinear oscillator. Though the nonlinear vibrations of the nonlinear oscillator alone under different nonlinear stiffness are significant, addition of the absorber having a relatively light mass to the nonlinear oscillator can greatly decrease amplitude of nonlinear vibrations. The vibration absorber is effective in attenuation of the nonlinear vibrations irrespective of the nonlinear stiffness of the weakly nonlinear oscillator. Figure $4 \mathrm{~d}$ shows the amplitude of vibrations of the nonlinear oscillator attached by absorber 
under different values of absorber mass. It is noted that for a given set of system parameters, increase of absorber mass results in an increase in amplitude in the neighbourhood of primary resonance frequencies. The two peaks in amplitude shift to the left with an increase of absorber mass. The main reason for this is that the natural frequency of absorber decreases with an increase of absorber mass, thereby leading to a small attenuation ratio and a shift in peak amplitude to the left.

The performance of vibration absorber on vibration suppression is also evident in the vibrational signals of the nonlinear oscillator with and without absorber, as shown in Figure 5 , for a combination of $k_{3}=2.5 \mathrm{~N} / \mathrm{m}, f_{1}=0.45 \mathrm{~N}, \Omega=2.13 \mathrm{rad} / \mathrm{s}$. The amplitude of vibration of the nonlinear primary oscillator with attached vibration absorber is much smaller than that of the nonlinear oscillator without absorber. On the contrary, the amplitude of vibration of the absorber is much higher than that of the nonlinear primary oscillator, indicating that the majority of vibrational energy of the nonlinear primary oscillator is transferred to the vibration absorber. This suggests the absorber can effectively suppress the primary resonance vibrations of the nonlinear oscillator.

\section{Conclusion}

The primary resonance response of a nonlinear oscillator can be suppressed by a linear vibration absorber which consists of a relatively light mass attached to the nonlinear oscillator by a linear damper and a linear spring. The small attachment of light mass can absorb vibrational energy without significantly modifying the nonlinear oscillator and adversely affecting its performance. The stiffness of the linked spring is much lower than the linear stiffness of the nonlinear oscillator itself. The contributions of the absorber stiffness and damping to the linear stiffness and damping of the nonlinear primary system can be considered as a perturbation. Thus the linearized natural frequencies of the nonlinear primary oscillator before and after addition of vibration absorber change only slightly. It is found that significant reduction of primary resonance vibrations can be achieved by using an absorber mass corresponding to $6 \%$ of the mass of the nonlinear oscillator and an absorber spring corresponding to $4.5 \%$ of the linear stiffness of the nonlinear oscillator. Saddle-node bifurcations and jump phenomena can also be eliminated through the application of a linear vibration absorber. The effects of the parameters of the mass-spring-damper absorber on the vibration suppression of the nonlinear oscillator have been studied. It has been found that a larger coupling damping results in a larger reduction of primary resonance 
vibrations. The ratio of two linearized natural frequencies of the resulting nonlinear system that is formed by the nonlinear primary system attached by the absorber is crucial for a higher desensitisation ratio and a lower attenuation ratio. The ratio of the two linearized natural frequencies is dependent on the absorber stiffness and mass. An increase of absorber stiffness will result in a larger ratio of the two linearized natural frequences however an increase of the absorber mass will decrease the ratio of the two linearized natural frequencies. It is suggested that the frequency ratio can be a certain value between 0.7 and 0.9 , which prevents the resultant two degree-of-freedom nonlinear system from one-to-one internal resonances and which can lead to a better performance of vibration reduction.

There are several distinct features in the suppression of the nonlinear vibrations of nonlinear systems using linear vibration absorber from the suppression of the vibrations of linear systems. For the nonlinear system considered in the present paper, due to its distinctive nature in primary resonance response from the dynamics of linear system, there is no need to shift the linearized natural frequency of the nonlinear primary system away from the excitation frequency, whereas the natural frequencies of the resulting system composed of the linear system attached by vibration absorber are generally tuned to be away from the excitation frequency. Furthermore, it is a common practice from the linear theory of vibration absorbers that the vibration absorber should be tuned to the resonance frequency of the linear primary system when suppressing the vibrations of linear systems. However, when using linear vibration absorber to suppress the nonlinear vibrations of nonlinear systems, the frequency of the absorber should not be tuned to the resonance frequency of the nonlinear primary system. This will avoid one-to-one internal resonances that otherwise may happen in the primary resonance response of the resultant nonlinear system. The presence of one-to-one internal resonances in a nonlinear system may result in instability of the periodic response and higher-amplitude quasi-periodic oscillations. Lastly, unlike the use of linear vibration absorber to suppress the vibrations of linear systems, there is no optimal value of the absorber damping for suppressing the nonlinear vibrations of nonlinear systems using linear vibration absorber.

Perturbation analysis suggested that the nonlinear vibrations of the nonlinear primary oscillator act as an external excitation to excite the vibrations of the absorber oscillator formed by the light mass. Most of the vibrational energy of the nonlinear primary 
oscillator is then transferred to the absorber through coupling spring and damper. The vibration absorber can effectively suppress the amplitude of oscillations of the nonlinear oscillator. Hence, by properly choosing the mass of absorber and stiffness of the linked spring and damping of the linked damper, the primary resonance response of the nonlinear oscillator can be reduced to a relatively small amplitude, while the excessive oscillatory energy is transferred to the small mass attachment. As such, the vibration absorber provides a promising alternative to the application of active vibration control of nonlinear system under conditions when active control is not feasible.

\section{Acknowledgments}

The authors would like to thank the anonymous reviewers for their helpful comments on the manuscript on which the quality of this work has been improved.

\section{References}

[1] A.H. Nayfeh, D.T. Mook, Nonlinear Oscillations, Wiley Interscience, New York, 1979.

[2] A. Maccari, Vibration amplitude control for a van der Pol-Duffing oscillator with time delay, Journal of Sound and Vibration 317 (2008) 20-29.

[3] S. Chatterjee, Vibration control by recursive time-delayed acceleration feedback, Journal of Sound and Vibration 317 (2008) 67-90.

[4] A. Maccari, Vibration control for the primary resonance of the van der Pol oscillator by a time delay state feedback, International Journal of Non-Linear Mechanics 38 (2003) 123-131.

[5] A. Maccari, Vibration control for the primary resonance of a cantilever beam by a time-delay state feedback, Journal of Sound and Vibration 259(2003) 241-251.

[6] C.Z. Qian, J.S. Tang, A time delay control for a nonlinear dynamic beam under moving load, Journal of Sound and Vibration 309 (2008) 1-8.

[7] J.C. Ji, Local bifurcation control of a forced single-degree-of-freedom nonlinear system: saddle-node bifurcation, Nonlinear Dynamics 25 (2001) 369-382.

[8] G. Chen, J.L.Moiola, H.O. Wang, Bifurcation control: theories, methods, and applications, International Journal of Bifurcation and Chaos 10 (2000) 511-548.

[9] A.Y.T. Leung, J.C. Ji, G.R.Chen, Resonance control for a forced single-degree-offreedom nonlinear system, International Journal of Bifurcation and Chaos 14(2004) 1423-1429. 
[10] S.S.Oueini, M.F. Golnaraghi, Experimental implementation of the internal resonance control strategy, Journal of Sound and Vibration 191(1996) 377-396.

[11] S.S. Oueini, A.H. Nayfeh, M.F. Golnaraghi, A theoretical and experimental implementation of a control method based on saturation, Nonlinear Dynamics 13 (1997) 189-202.

[12] P.F. Pai, B. Rommel, M.J.Schulz, Non-linear vibration absorbers using higher order internal resonances, Journal of Sound and Vibration 234 (2000) 799-817.

[13] D.J. Mead, Passive Vibration Control, John Wiley \& Sons Ltd, Chichester, West Sussex, England, 2000.

[14] J.B. Hunt, Dynamic Vibration Absorbers, London: Mechanical Engineering Publications, 1979.

[15] B. G. Korenev, and L.M. Reznikov, Dynamic Vibration Absorbers, Wiley, 1993.

[16] D.J. Inman, Engineering Vibration, 2nd ed., Prentice Hall, Englewood Cliffs, NJ, 2001.

[17] S.S. Rao, Mechanical Vibration, 4th ed., Person Prentice Hall, New Jersey, 2001.

[18] K.F. Liu, J. Liu, The damped dynamic vibration absorbers: revisited and new result. Journal of Sound and Vibration 284 (2005) 1181-1189.

[19] Y. Du, R.A. Burdisso, E. Nikolaidis, Control of internal resonances in vibration isolators using passive and hybrid dynamic vibration absorbers. Journal of Sound and Vibration 286 (2005) 697-727.

[20] Y.L. Cheung, W.O. Wong, $H_{\infty}$ and $H_{2}$ optimisation of a dynamic vibration absorber for suppressing vibrations in plates. Journal of Sound and VIbraiton 320 (2009) 29-42.

[21] J.B. Hunt, J.C. Nissen, The broadband dynamic vibration absorber. Journal of Sound and Vibration 83 (1982) 573-578.

[22] J.C. Nissen, K. Popp, B. Schmalhorst, Optimization of a nonlinear dynamic vibration absorber. Journal of Sound and Vibration 99(1985) 149-154.

[23] H.J. Rice, J.R. McCraith, On practical implementations of the nonlinear vibration absorber. Journal of Sound and Vibration 110 (1986) 161-163.

[24] I.N. Jordanov, B.I. Cheshankov, Optimal design of linear and nonlinear dynamic vibration absorbers. Journal of Sound and Vibration 123 (1988) 157-170.

[25] C.W. Bert, D.M. Egle, D.J. Wilkins, Optimal design of a nonlinear dynamic absorber. Journal of Sound and Vibration 137 (1990) 347-352.

[26] H.J. Rice, Combinational instability of the non-linear vibration absorber. Journal of Sound and Vibration 108 (1986) 526-532. 
[27] J. Shaw, S.W. Shaw, A.G. Haddow, On the response of the nonlinear vibration absorber. International Journal Non-Linear Mechanics 24 (1989) 281-293.

[28] S. Natsiavas, Steady state oscillations and stability of non-linear dynamic vibration absorbers. Journal of Sound and Vibration 156 (1992) 227-245.

[29] J.H. Bonsel, R.H.B. Fey, H. Nijmeijer, Application of a dynamic vibration absorber to a piecewise linear beam system. Nonlinear Dynamics 37 (2004) 227-243. 


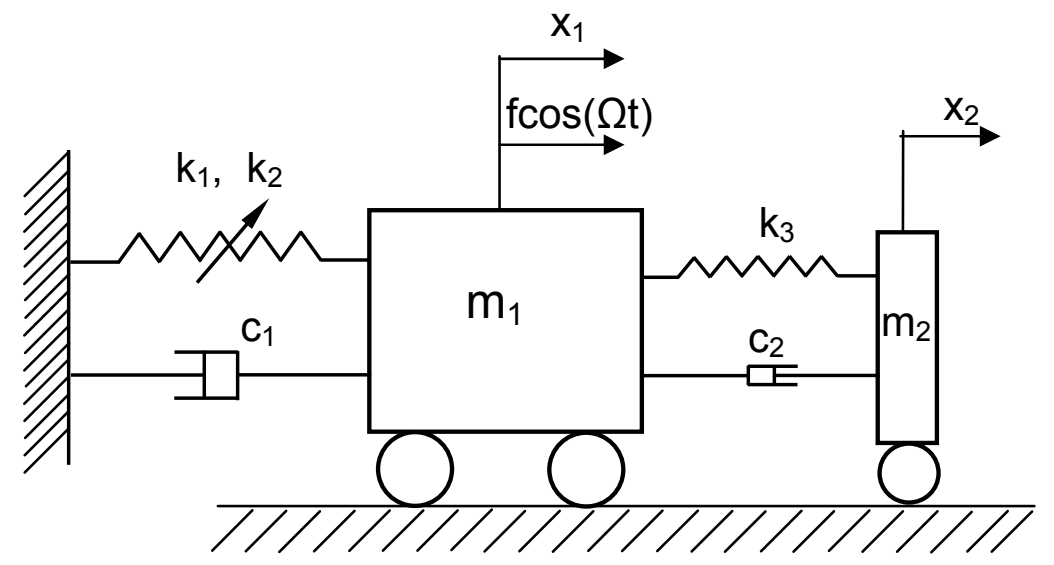

Figure 1. The combined two degree-of-freedom nonlinear system composed of nonlinear primary oscillator and linear vibration absorber. 

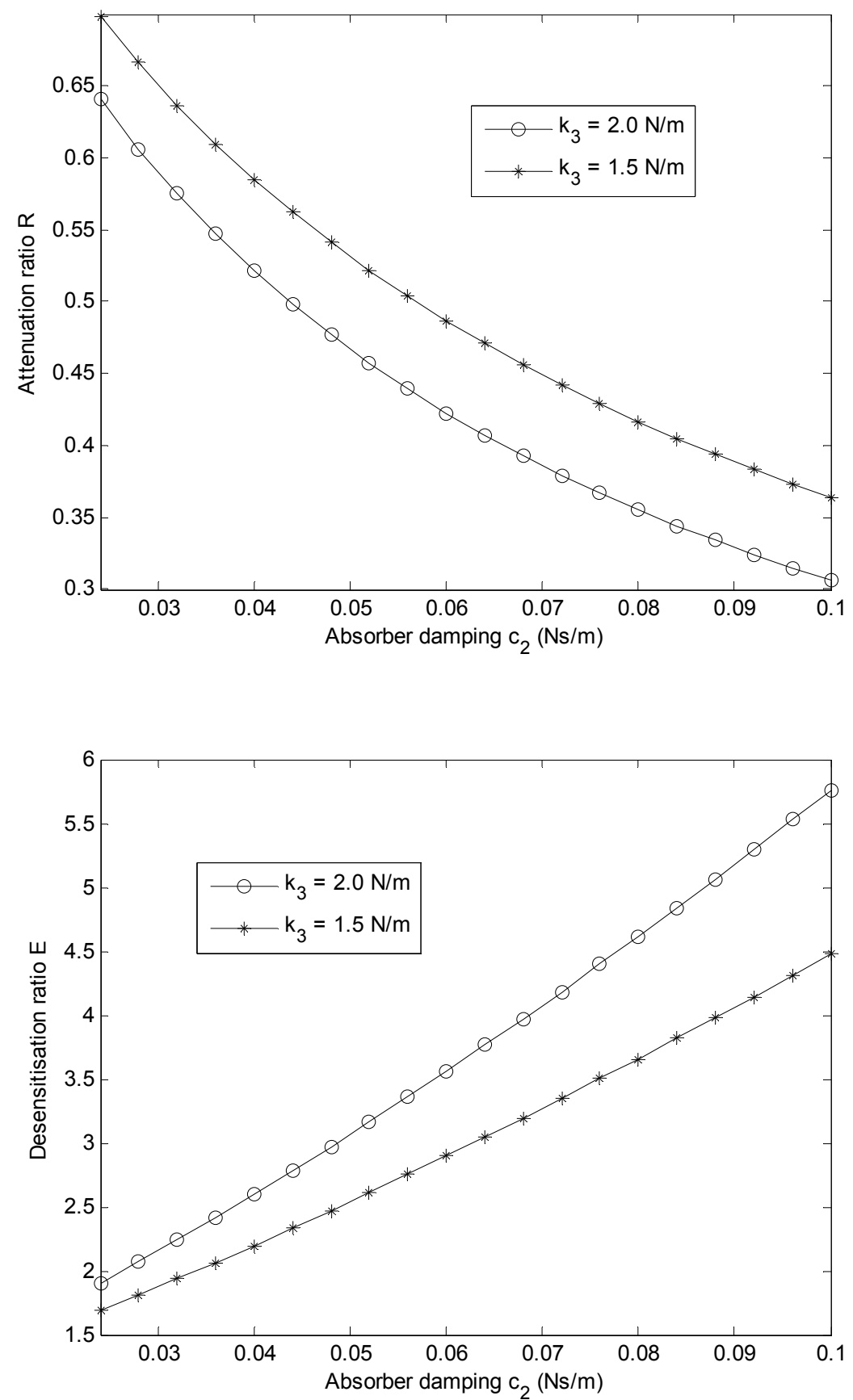

Figure 2 (a) 

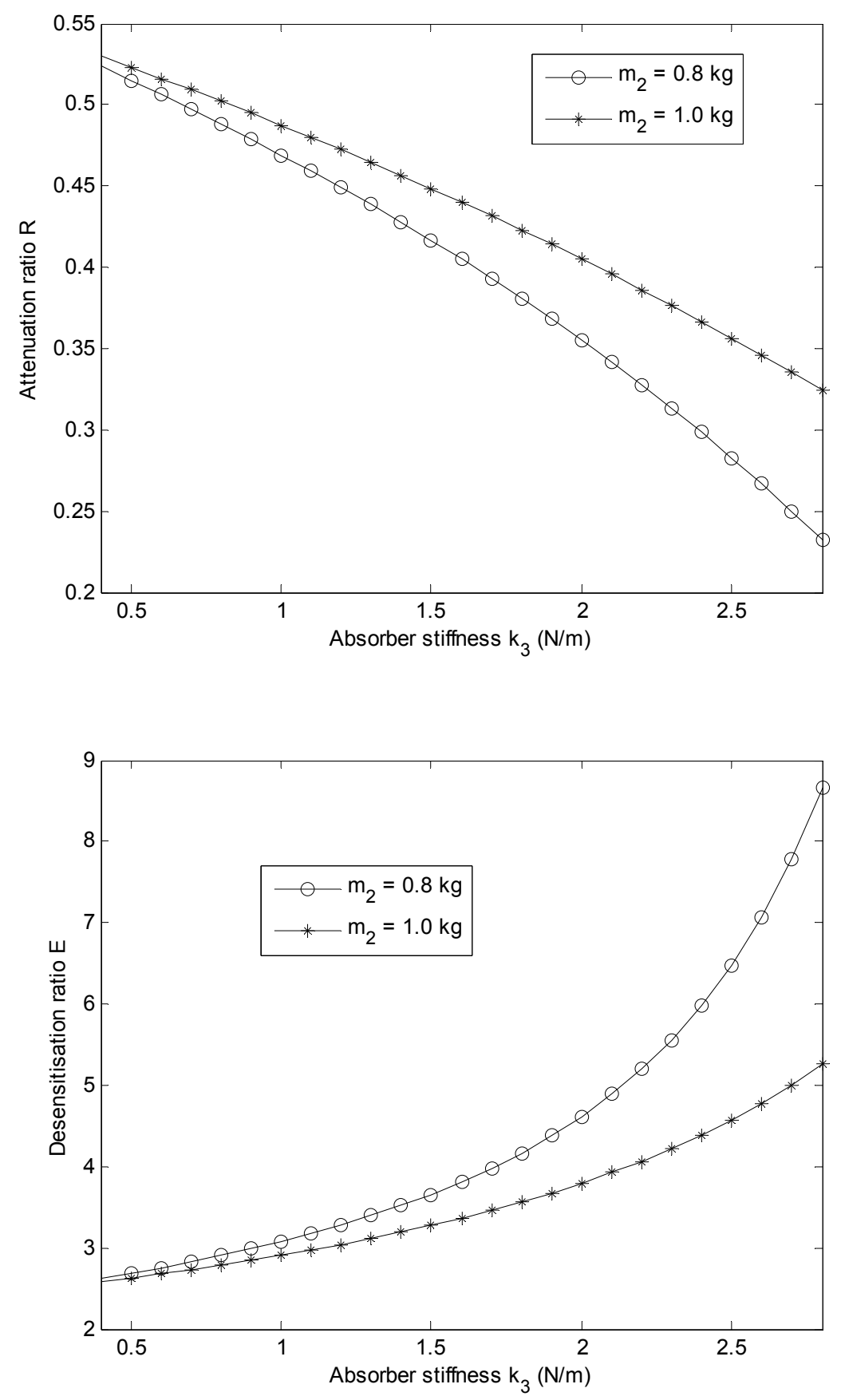

Figure 2 (b) 

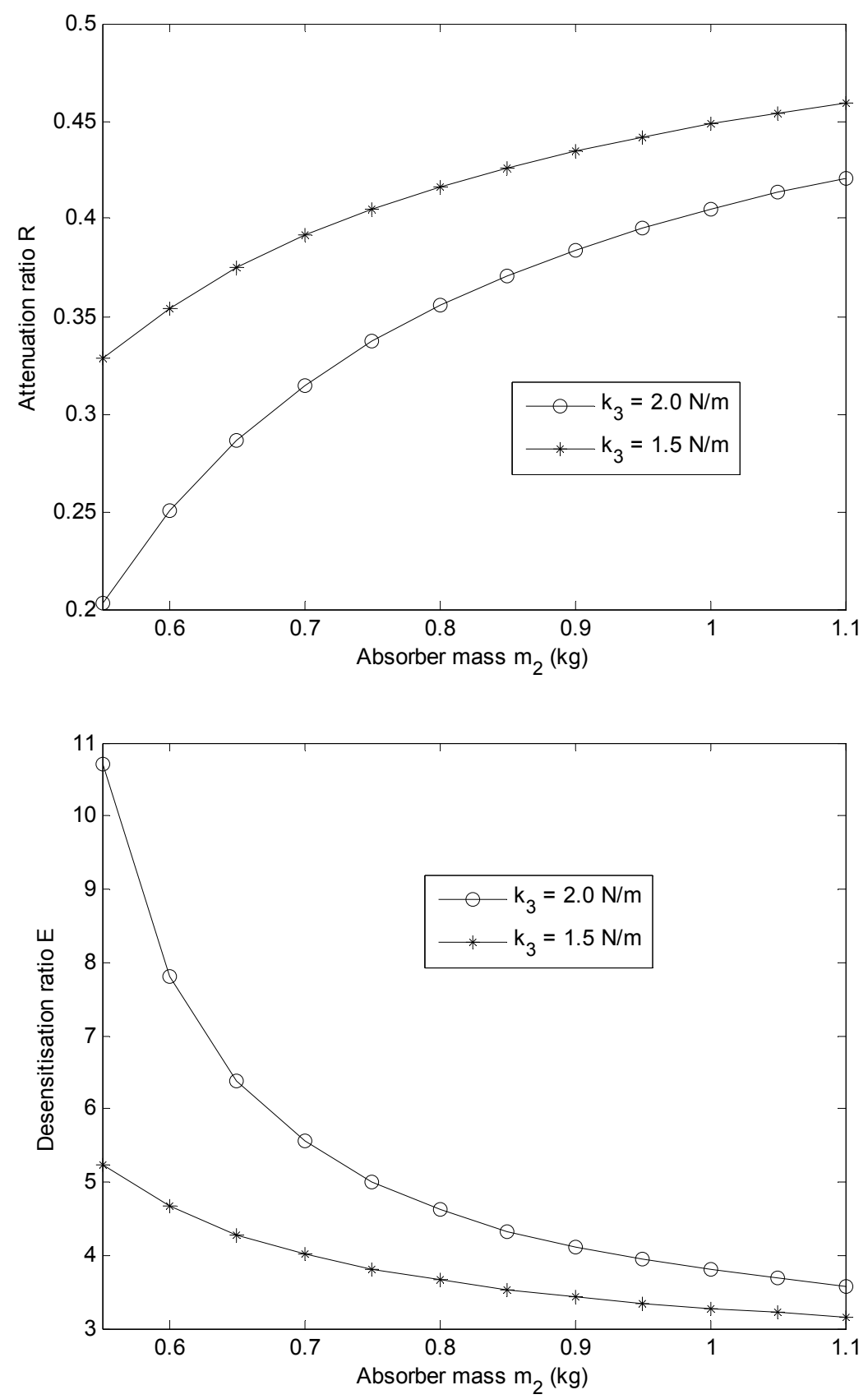

Figure 2 (c)

Figure 2: Variations of attenuation ratio and desensitisation ratio with damping, stiffness and mass of the absorber; (a) with absorber damping $c_{2}$, (b) with absorber stiffness $k_{3}$, (c) with absorber mass $m_{2}$. 


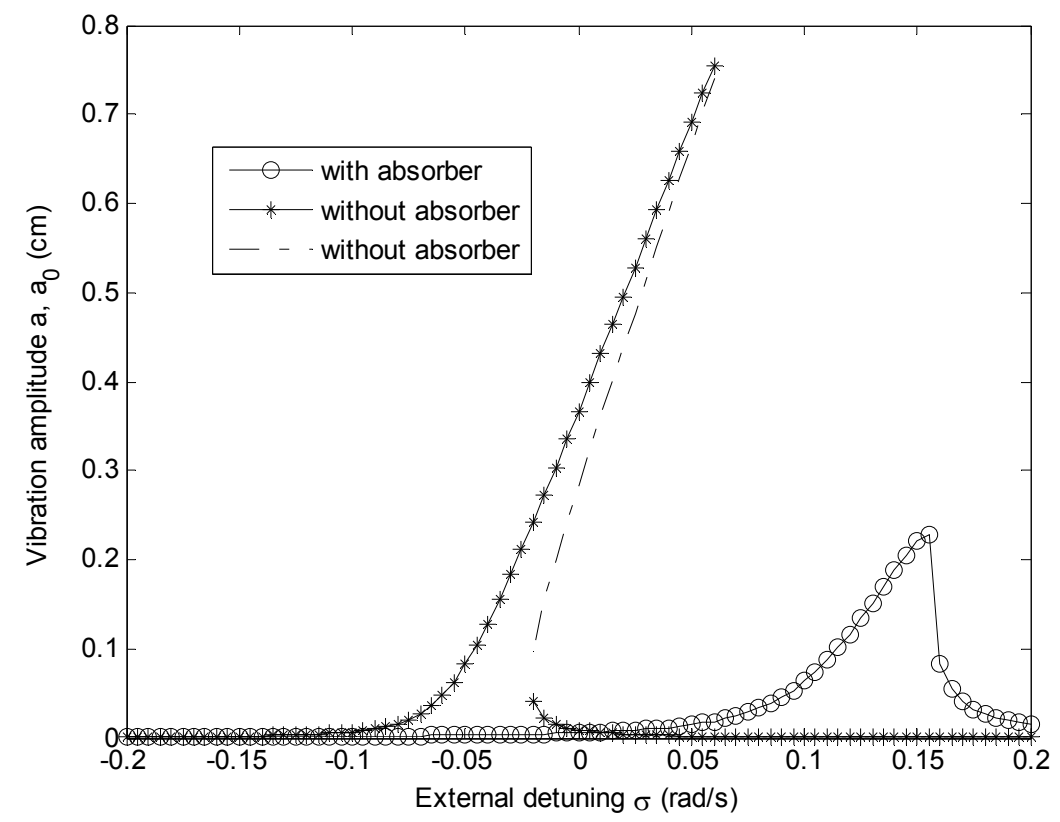

Figure 3 The frequency-response curves of the nonlinear primary oscillator before and after vibration absorber is attached. Solid lines denote stable solutions and dash-dot line represents unstable solutions. 


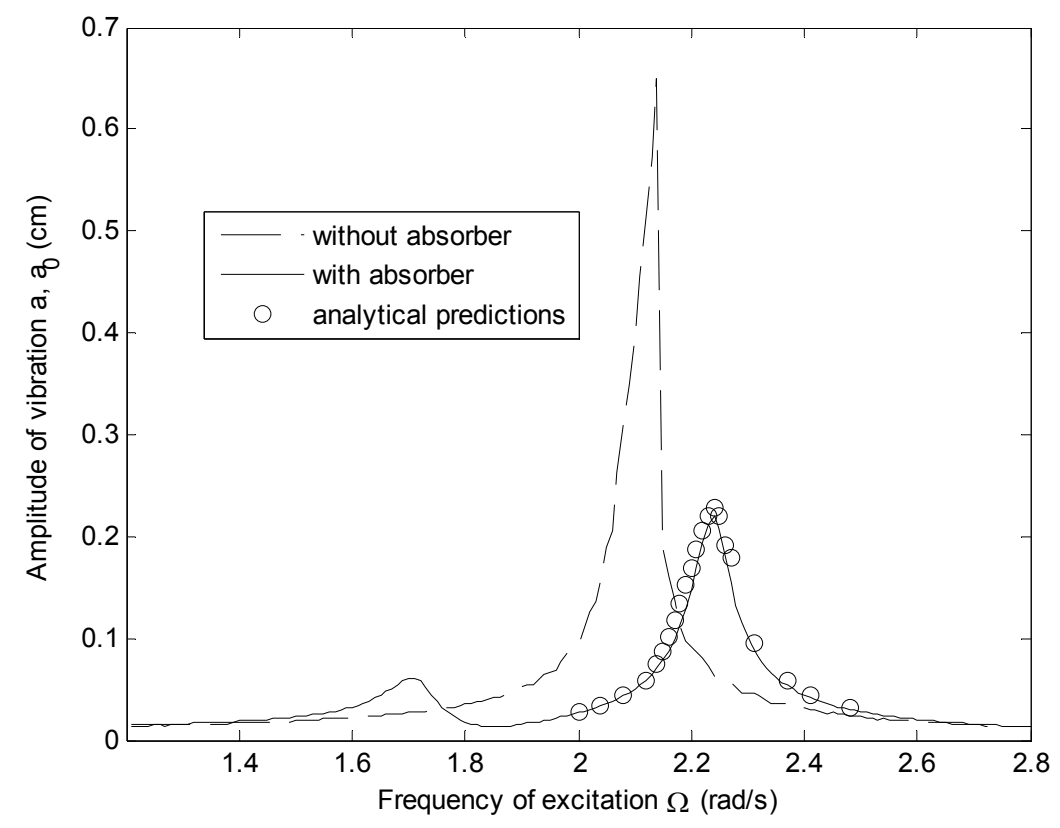

Figure 4 (a)

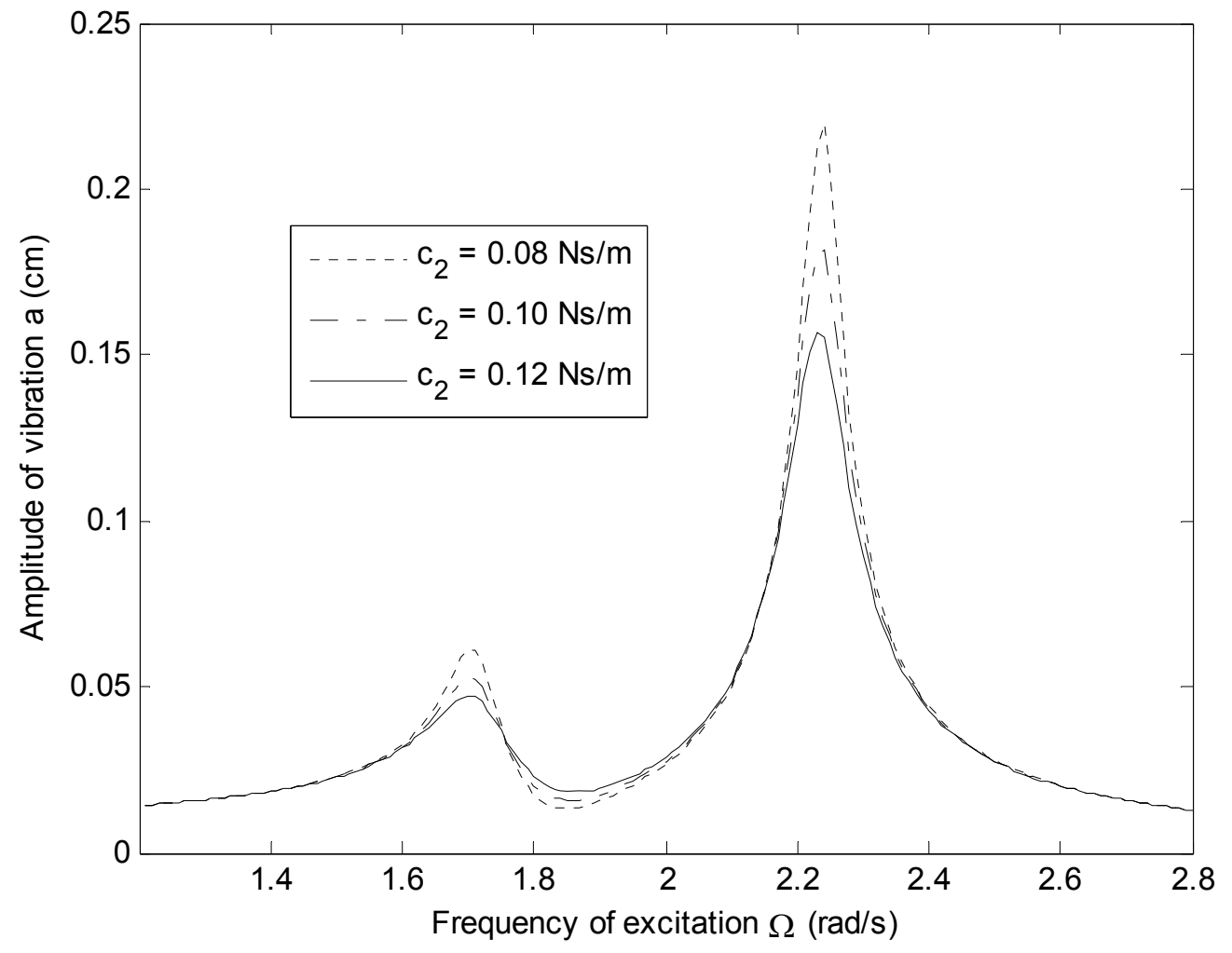

Figure 4 (b) 


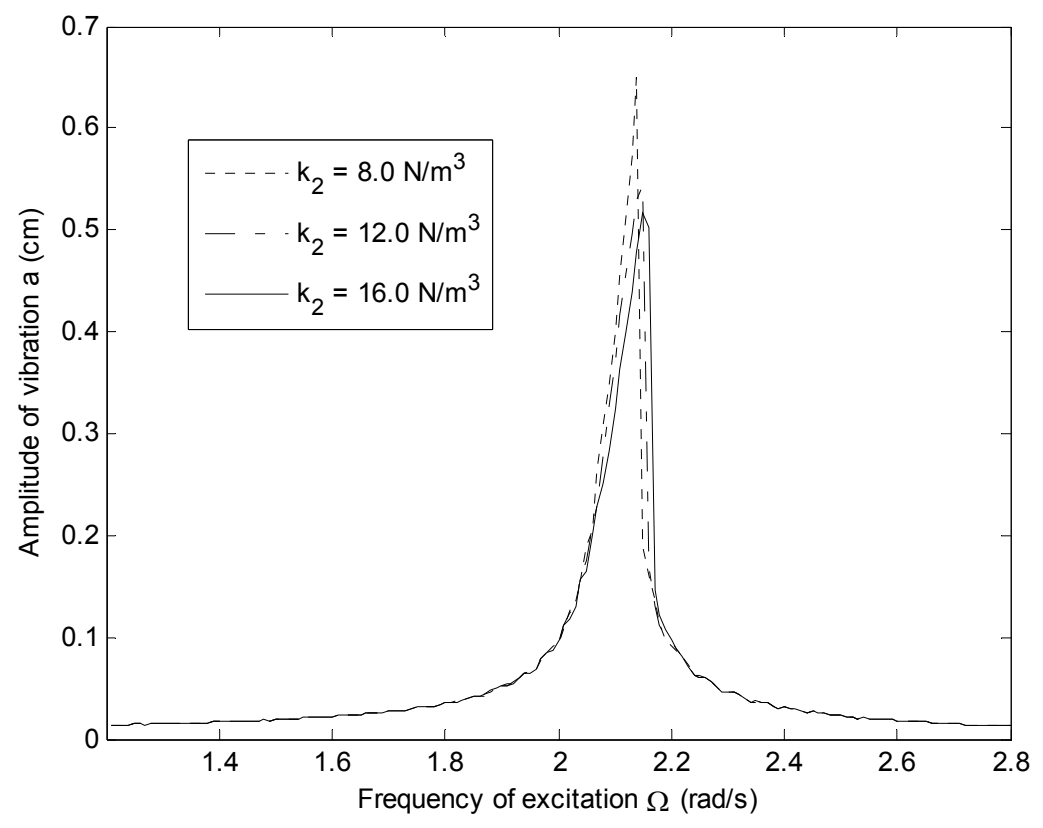

Figure 4 (c)

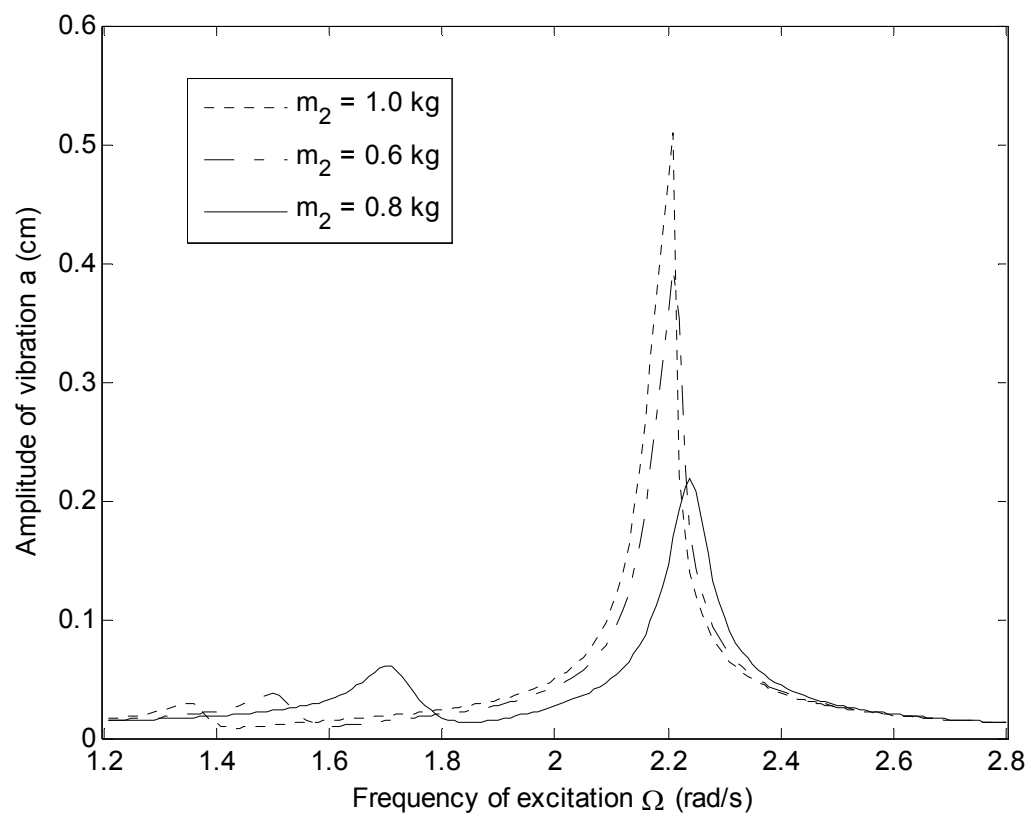

Figure $4(d)$

Figure 4: Frequency-response curves of the nonlinear oscillator in a large interval of the frequency of excitation. 


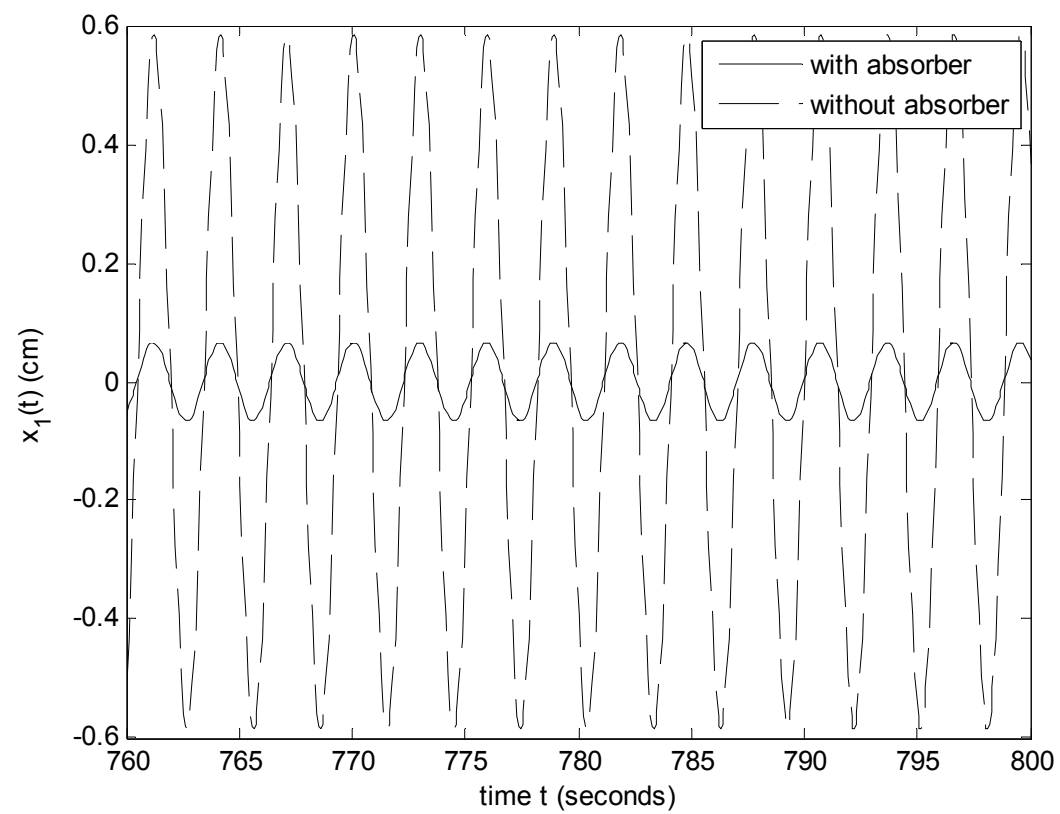

Figure 5 (a)

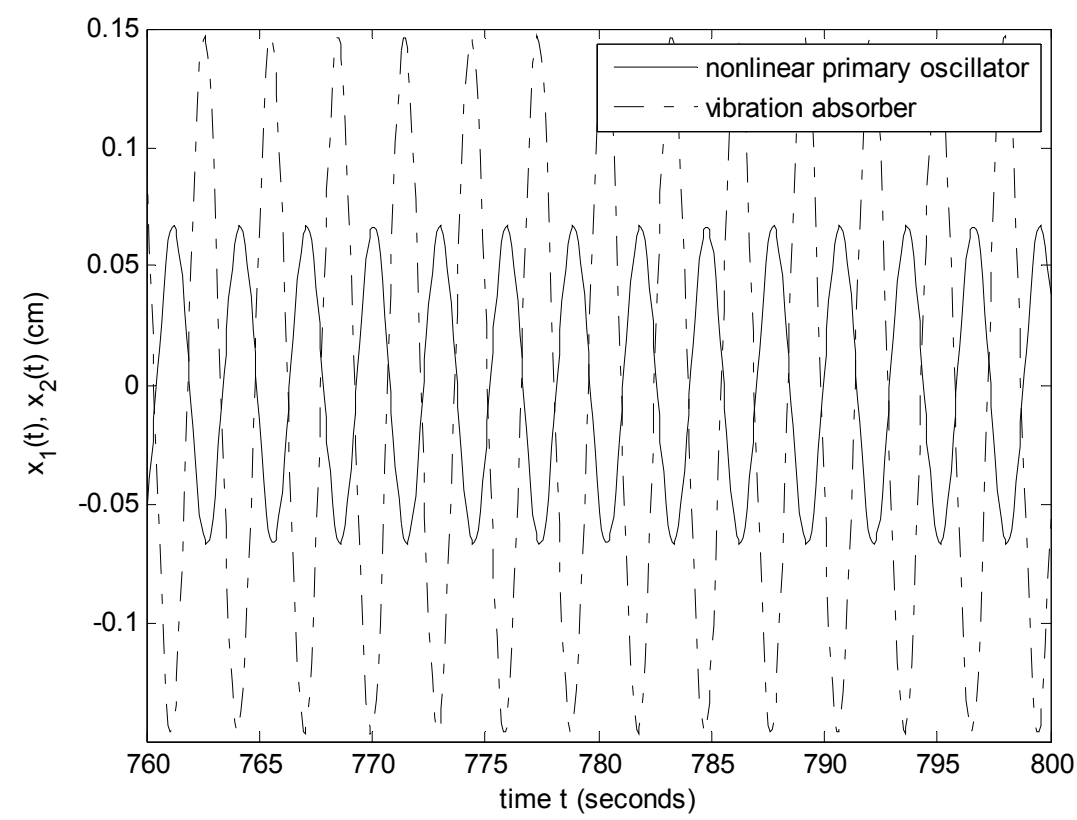

Figure 5 (b)

Figure 5: The time histories of primary resonance response of the nonlinear primary oscillator and vibration absorber for the combination of $k_{3}=2.5 \mathrm{~N} / \mathrm{m}, f_{1}=0.45 \mathrm{~N}, \Omega=2.13 \mathrm{rad} / \mathrm{s}$; a) nonlinear primary system before and after vibration absorber is attached, b) nonlinear primary system and vibration absorber after vibration absorber is attached to the nonlinear primary oscillator. 